\title{
Prediction of pulse-to-pulse intensity fluctuation characteristics of high power ultrafast fiber amplifiers
}

K. Gürel, P. Elahi, L. Budunoğlu, Ç. Şenel, P. Paltani, and F. Ö. Ilday

Citation: Appl. Phys. Lett. 105, 011111 (2014);

View online: https://doi.org/10.1063/1.4888298

View Table of Contents: http://aip.scitation.org/toc/apl/105/1

Published by the American Institute of Physics

\section{Articles you may be interested in}

Invited Article: A compact optically coherent fiber frequency comb

Review of Scientific Instruments 86, 081301 (2015); 10.1063/1.4928163

High-power ultrafast Yb:fiber laser frequency combs using commercially available components and basic fiber tools

Review of Scientific Instruments 87, 093114 (2016); 10.1063/1.4962867

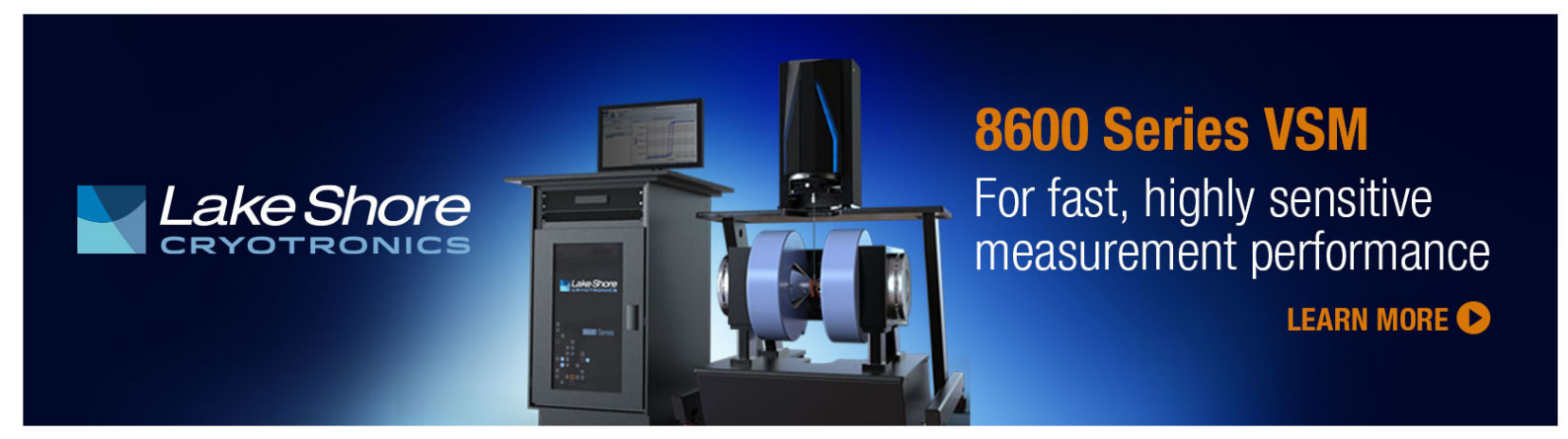




\title{
Prediction of pulse-to-pulse intensity fluctuation characteristics of high power ultrafast fiber amplifiers
}

\author{
K. Gürel, ${ }^{1}$ P. Elahi, ${ }^{1, a)}$ L. Budunoğlu, ${ }^{1}$ C. S. Şenel,,${ }^{1,2}$ P. Paltani, ${ }^{1}$ and F. Ö. Ilday ${ }^{1,3}$ \\ ${ }^{1}$ Department of Physics, Bilkent University, TR-06800 Ankara, Turkey \\ ${ }^{2}$ TUBITAK National Metrology Institute (UME), 41470, Kocaeli, Turkey \\ ${ }^{3}$ Department of Electrical and Electronics Engineering, Bilkent University, TR-06800 Ankara, Turkey
}

(Received 7 April 2014; accepted 26 June 2014; published online 8 July 2014)

\begin{abstract}
We report on the experimental characterization and theoretical prediction of pulse-to-pulse intensity fluctuations, namely, intensity noise, for ultrafast fiber amplifiers. We present a theoretical model with which the intensity noise of a Yb-doped fiber amplifier can be predicted with high accuracy, taking into account seed and pump noise, as well as generation of amplified spontaneous emission. Transfer of pump and seed signal modulations to the amplified output during fiber amplification are investigated thoroughly. Practically, our model enables design and optimization of fiber amplifiers with regards to their intensity noise performance. As a route to reducing noise imparted by pump diodes in a double-clad amplifier, we show the use of multiple, low-power diodes is more beneficial compared to a single, high-power diode due to the largely uncorrelated nature of their individual noise contributions. (C) 2014 AIP Publishing LLC. [http://dx.doi.org/10.1063/1.4888298]
\end{abstract}

Rapid progress is being reported on the development of high-power fiber laser-amplifier systems. However, despite its importance in various applications, intensity noise of fiber amplifiers has not received a close scrutiny, particularly for high-power amplifiers. We reported a systematic study of intensity noise of passively mode-locked fiber oscillators, operating in different mode-locking regimes. ${ }^{1}$ A key conclusion of this study was that there is often a trade-off between the noise level of the oscillator and the highest pulse energy that can be extracted. Thus, the route to low-noise highpower operation could be to operate the oscillator at moderate power levels and amplify externally. A major contributor to noise during amplification is transfer of noise of the pump lasers, which is particularly high for high-power multimode pump lasers. ${ }^{2}$ In addition, there are various applications where modulations of the signal or the pump need to be transferred to the amplified signal or an existing modulation to be preserved, such as carrier-envelope phase (CEP) stabilization, ${ }^{3}$ where pump noise is an important factor.

Here, we follow a simple, yet accurate theoretical model $^{4-6}$ for calculation of transfer of fluctuations in intensity (intensity noise) present in the seed and pump signals to the output of a fiber amplifier, and obtain noise transfer functions (NTF). The predictions of the model are then compared with and validated by experimental results obtained from low-power Yb-fiber amplifiers, which are pumped through the core. Then, we apply our model to predict the output noise performance of high-power amplifiers pumped by multimode pump diodes, which have substantially higher intensity noise. The physics of noise transfer is identical for both amplifier systems, but important technical differences exist due to the use of single- and multi-mode pump sources, respectively. It is found that the relatively much higher power fluctuations of multimode pump diodes end up dominating the amplifier performance. As a route to passively

\footnotetext{
${ }^{a)}$ Electronic mail: pelahi@fen.bilkent.edu.tr
}

decreasing the noise imparted by multi-mode diodes in cladding-pumped amplifiers, we evaluate the impact, in terms of the noise performance, of using multiple, lowpower pump diodes as opposed to a single, high-power diode.

We decouple the fast and slow dynamics governing the amplification of broadband pulses in a fiber amplifier as follows: The fast dynamics is modeled by a generalized nonlinear Schrödinger equation as in Ref. 7, using an effective gain model. The gain parameters are determined from a more accurate model based on Ref. 8, where the signal power is interpreted as the average power of the pulse train. This is justified as long as the repetition rate is in the $\mathrm{MHz}$ range or higher and no individual pulse is energetic enough to saturate the amplifier. We use a generalized version of this wellknown model to include modulations (as well as noise) of the seed and the pump; this constitutes the slow-dynamics part of our model. The signal and pump modulations and population levels at a specific frequency of $\omega=2 \pi \nu$ are described by

$$
\begin{gathered}
I_{p(s)}(z, t)=I_{p(s), 0}(z)\left(1+q_{p(s)}(z, \omega) e^{i \omega t}\right)+c . c ., \\
n_{1(2)}(z, t)=n_{1(2), 0}(z)\left(1+q_{1(2)}(z, \omega) e^{i \omega t}\right)+c . c .
\end{gathered}
$$

where $I_{p(s)}(z, t)$ are the pump (signal) intensities and $n_{1(2)}(z, t)$ are the fractional population densities of the lower (upper) states, and $q(\omega, z)$ represent the modulation amplitudes; c.c. denotes complex conjugate. $I_{p(s), 0}(z)$ and $n_{1(2), 0}(z)$ correspond to solutions without modulation. $z$ denotes position along the gain fiber of total length $L$. Inserting Eqs. (1) and (2) into the coupled equations for population densities and power distribution ${ }^{9}$ yields

$$
\begin{gathered}
I_{p(s), 0}(z) \frac{d q_{p(s)}(z, \omega)}{d z}-f_{p(s)}(z) X_{p(s)}(z, \omega) q_{p(s)}(z, \omega) \\
-f_{s(p)}(z) X_{s(p)}(z, \omega) q_{s(p)}(z, \omega)+c . c .=0,
\end{gathered}
$$


where $\sigma_{12}^{p(s)}$ and $\sigma_{21}^{p(s)}$ denote the absorption and emission cross-sections, respectively, for pump (signal), and

$$
\begin{gathered}
f_{p(s)}(z)=\left[\frac{\sigma_{12}^{p(s)} n_{1,0}(z)-\sigma_{21}^{p(s)} n_{2,0}(z)}{h \nu_{p(s)}}\right] I_{p(s), 0}(z), \\
X_{p(s)}(z, \omega)=A_{p(s)}(z) /(B(z)+i \omega), \\
B(z)=\frac{\left(\sigma_{12}^{p}+\sigma_{21}^{p}\right)}{h \nu_{p}} I_{p, 0}(z)+\frac{\left(\sigma_{12}^{s}+\sigma_{21}^{s}\right)}{h \nu_{s}} I_{s, 0}(z)+\frac{1}{\tau}, \\
A_{p(s)}=\Gamma_{p(s)}\left[\sigma_{12}^{p(s)}+\sigma_{21}^{p(s)}\right] I_{p(s), 0}(z),
\end{gathered}
$$

$\nu_{p(s)}$ is pump (signal) photon energy, $\tau$ is gain relaxation time, $\Gamma_{p(s)}$ is pump (signal) overlap factor. Equation (3) along with Eqs. (4) and (5) can be solved numerically following Ref. 8. The modulation response functions, $\xi_{p}(\nu)$ for pump and $\xi_{s}(\nu)$ for signal are given by

$$
\xi_{p(s)}(\nu)=q_{s}(L, \nu) / q_{p(s)}(0, \nu),
$$

which are essentially Lorentzian-like in shape, as discussed below. This approach can be generalized trivially, if more than a single frequency is involved.

The schematic of our experimental setup is shown in Fig. 1. A similariton Yb-doped fiber laser ${ }^{10}$ provides the seed pulses. We utilize two different amplifiers, a corepumped, low-power $(<1 \mathrm{~W})$ amplifier with a single-mode diode laser as pump, similar to that in Ref. 10 and a cladding-pumped, high-power (up to $50 \mathrm{~W}$ ) amplifier with multiple multi-mode diode lasers as pump. In the latter case, additional intermediate amplifier stages are present between the amplifier under characterization and the seed oscillator, which is not shown in the figure. The measurement and characterization setup is common for both cases. The seed signal traverses a fiber-coupled acousto-optic modulator (AOM), after which it is split by a fiber coupler, where one port is directed to a high-resolution power meter (not shown in the figure) or a low-noise baseband analyzer (UPV, Rohde \& Schwartz) for analysis and the other port seeds the Yb-doped amplifier.

We employ the core-pumped, low-power amplifier for verification of the model due to its ease of experimentation. ${ }^{11}$ The amplifier comprises of a $120 \mathrm{~cm}$-long Yb-doped fiber. The amplifier is forward-pumped through a wavelength division multiplexer (WDM) by a single-mode pump diode operating at a central wavelength near $976 \mathrm{~nm}$. A portion of the output of the pump diode and that of the amplifier output are

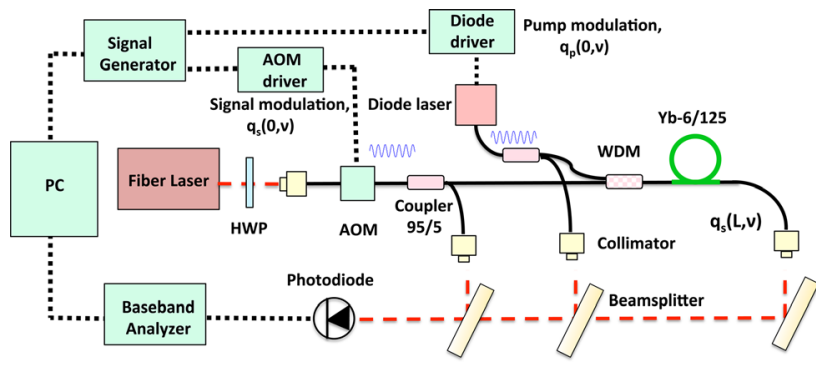

FIG. 1. Schematic setup of the intensity noise measurement. AOM: acoustooptic modulator, HWP: half-wave-plates, and WDM: wavelength division multiplexer. directed to the power meter or the baseband analyzer where the relative intensity noise (RIN) is measured. The amplified signal is separated from any unabsorbed pump light using a second WDM. The NTFs are measured by modulating the pump and seed powers by directly modulating the pump diode's current and through the AOM, respectively, over the frequency range of $1 \mathrm{~Hz}-250 \mathrm{kHz}$, where the upper limit is set by the analyzer. The NTF is obtained via the same method as in Ref. 12. However, the entire measurement is automated via a computer.

Measured and calculated NTF's for the case of $1 \mathrm{~mW}$ of seed and $100 \mathrm{~mW}$ of pump power are illustrated in Fig. 2(a). The NTF's are fit very well by generalized Lorentzian functions, given by $\xi_{p}(\nu)=\xi_{p, 0} /\left(1+\left(\nu / \nu_{p, 0}\right)^{n}\right)$ for the pump and $\xi_{s}(\nu)=1-\left(1-\xi_{s, 0}\right) /\left(1+\left(\nu / \nu_{s, 0}\right)^{n}\right)$ for the seed. Here, $\xi_{p, 0}$ and $\xi_{s, 0}$ represent the small frequency transfer coefficients and, $\nu_{p}$ and $\nu_{s}$ are the $3-\mathrm{dB}$ cutoff frequencies for pump and signal, respectively. A prerequisite for using the transfer function approach is that the system under study must be linear. Indeed, the small-frequency transfer coefficients, $\xi_{p, 0}$ and $\xi_{s, 0}$, are verified to be independent of the modulation depth over a large range of seed power to pump power ratios (Fig. 2(b)). $\xi_{p, 0}$ and $\xi_{s, 0}$, and the 3-dB cutoff frequencies, $\nu_{p, 0}$ and $\nu_{s, 0}$ on the ratio of the launched seed power to the pump power are shown in Figs. 2(c) and 2(d). Importantly, the transfer coefficients for pump and seed always approach 0 and 1 , respectively, at high frequencies, meaning that high-frequency pump noise has practically no influence on amplifier performance. Conversely, any seed modulations are strongly suppressed at low frequencies due to the saturation effect. As the signal and pump NTF's are complementary, rendering possible broadband modulation (e.g., for CEP stabilization) by modulating both the seed and the pump at high and low frequencies, respectively.

As an application, we utilize the theoretical model to predict amplifier intensity noise using the leading noise sources, which are the RIN present on the seed signal, the contribution of the pump laser noise and excess quantum noise due to amplified spontaneous emission (ASE). Nominally, a full
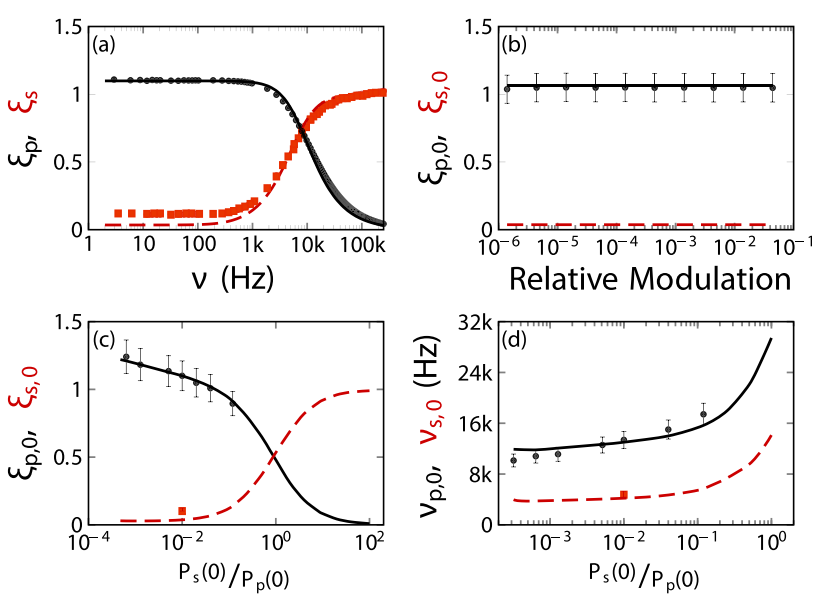

FIG. 2. Measured (circles for pump and squares for signal) and calculated (solid line for pump and dashed line for signal) (a) noise transfer function, NTF vs. modulation frequency, (b) $\xi_{p, 0}$ and $\xi_{s, 0}$ vs. modulation depth, (c) $\xi_{p, 0}$ and $\xi_{s, 0}$ vs. seed-pump power ratio, and (d) $\nu_{p, 0}$ and $\nu_{s, 0}$ vs. seed-pump power ratio. 
calculation of combined effect of these noise processes is extremely demanding in terms of computation time, as one requires an integration time at least as long as the inverse of the lowest frequency of interest. Most of the pump- and environment-induced noise appears at low frequencies (typically, few $\mathrm{kHz}$ or less). In addition, in order to obtain statistically meaningful information, multiple calculations would be needed. Our approach, however, is based on using the NTF of signal and pump to determine the average RIN to be imparted at each frequency on the amplifier output based on the RIN value of the signal and the pump at that frequency. We treat noise due to signal or pump and quantum fluctuations to be additive in nature. This approach promptly ignores any interaction between these noise sources.

We inherently assume that all noise sources introduce weak enough fluctuations that they can be treated as firstorder perturbations. The average relative intensity noise of the amplifier output is given by

$$
\begin{aligned}
\overline{I_{\mathrm{amp}}^{2}}(\nu)= & \eta_{1}\left[\overline{I_{\mathrm{s}}^{2}}(\nu) \xi_{s}^{2}(\nu)+\overline{I_{\mathrm{p}}^{2}}(\nu) \xi_{p}^{2}(\nu)\right] \\
& +\eta_{2}\left[\overline{I_{\text {ase }}^{2}}(\nu)+\overline{I_{\mathrm{p}}^{2}}(\nu) \xi_{a}^{2}(\nu)\right] .
\end{aligned}
$$

Here, $\eta_{1}$ and $\eta_{2}$ represent the fractional power of coherent (amplified signal) and incoherent (ASE) portions of the amplifier output, respectively. $\xi_{a}(\nu)$ is the pump-to-ASE NTF, which has the same form as $\xi_{p}(\nu)$ with correspondingly different parameters. We note that $\xi_{p}^{2}(\nu)$ is exactly a Lorentzian. $\overline{I_{\mathrm{s}}^{2}}(\nu)$ and $\overline{I_{\mathrm{p}}^{2}}(\nu)$ represent the RIN spectra of the seed and pump signals. The quantum fluctuations constitute white noise given by

$$
\overline{I_{\text {ase }}^{2}}(\nu)=\Delta \nu_{\mathrm{opt}}^{-1}
$$

where $\Delta \nu_{\text {opt }}$ is the optical bandwidth of the ASE spectrum. ${ }^{13}$

The pump and seed RIN contributions, weighted by their respective transfer functions, and RIN due to ASE are all added up linearly, which ignores any interaction between these noise sources. This is justified as long as the noise levels are low. As an example, Fig. 3(a) shows the measured RIN of
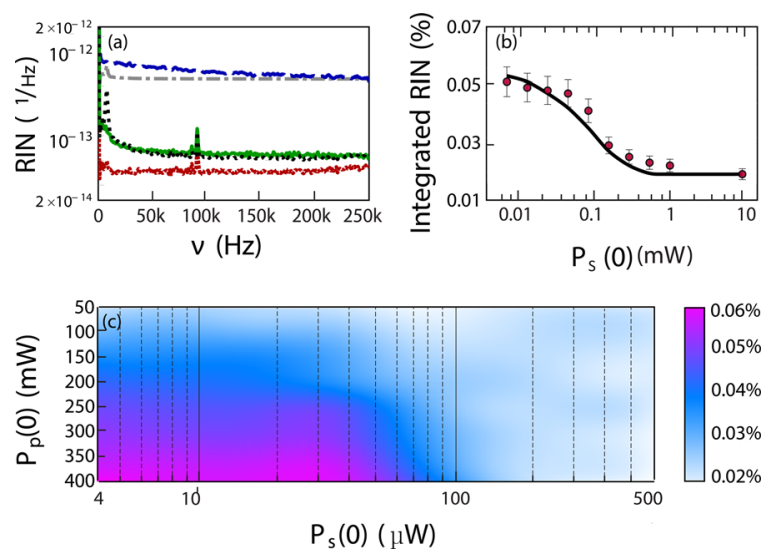

FIG. 3. (a) Measured RIN spectra of the seed (red-densely dotted curve), ASE (blue-dashed curve), amplifier output (green-solid curve), calculated RIN spectra of ASE (gray-dotted dashed curve), and amplifier output (blackloosely dotted curve) for core-pumped amplifier. (b) Measured (dots) and calculated (solid curve) integrated RIN values as a function of signal power for low power, single-clad amplifier. (c) Semi-log contour plot of integrated RIN for varying seed and pump powers. the seed, pump, amplifier output (seed and pump powers of 12 $\mathrm{mW}$ and $100 \mathrm{~mW}$, respectively), as well as pure ASE (no seed). Calculated RIN for strong seed and ASE reveal very good agreement with the experimental results. The integrated (from $1 \mathrm{kHz}$ to $250 \mathrm{kHz}$ ) RIN values for the single-clad amplifier output are measured and calculated to be $0.0192 \% \pm 0.0005$ and $0.0190 \% \pm 0.0005$, respectively.

The integrated RIN is observed to decrease rapidly with increasing seed under constant pump power, which are shown by experiment and verified by theoretical model (Fig. 3(b)). For additional insight, we characterize the RIN of fiber-amplified pulses as a function of both seed and pump powers (Fig. 3(c)). The general behavior is as to be expected: The combination of low seed and high pump powers leads to excessive ASE generation, which increases the RIN level. We note that for a similar experiment with seed wavelength of $1050 \mathrm{~nm}, 4$ times higher seed power is required compared to seeding at $1030 \mathrm{~nm}$. Stronger seed is required to saturate the amplifier at $1050 \mathrm{~nm}$, compared to $1030 \mathrm{~nm}$, leading to less ASE generation, due to the lower transition cross section of the Yb-doped fiber at the former wavelength.

In order to analyze the case of a high-power amplifier, similar noise characterizations have been made for a claddingpumped amplifier consisting of a multi-port pump-signal combiner (MPC) with 6 pump ports and 1 signal port, connected to a low-doped double-clad (DC) Yb-fiber, followed by a high-doped Yb-fiber. ${ }^{14}$ The low-doped segment is $1.8 \mathrm{~m}$ long with $6.5 \mathrm{~dB} / \mathrm{m}$ absorption at $980 \mathrm{~nm}$, and the high-doped segment is $1.6 \mathrm{~m}$ long with $10.8 \mathrm{~dB} / \mathrm{m}$ absorption at $980 \mathrm{~nm}$. Both fibers have a core diameter of $25 \mu \mathrm{m}$, core numerical aperture of 0.08 , cladding diameter of $250 \mu \mathrm{m}$, which enables very low-loss splicing between them. We employ two $25-\mathrm{W}$ diodes and three $32-\mathrm{W}$ diodes, all operating at $976 \mathrm{~nm}$, for pumping. The noise of combination of multimode diodes is characterized at the output of the MPC. The $25 \mathrm{~W}$ diodes are turned on or off accordingly. Fig. 4(a) shows the RIN of pump and amplifier output at $1.5 \mathrm{~W}$ of seed and $72 \mathrm{~W}$ pump powers. The integrated RIN values are measured and calculated to be $1.42 \% \pm 0.05$ and $2.00 \% \pm 0.05$, respectively. The integrated RIN is observed to stay constant over the output power range of $5 \mathrm{~W}$ to $50 \mathrm{~W}$ with seed power of $1.5 \mathrm{~W}$ (Fig. 4(b)).

Additionally, an experiment has been done to demonstrate the averaging of intensity noise over multiple pump diodes with incoherent noise profile (Fig. 5). We compared the use of a single, high-power pump diode to that of a varying number of pump diodes operated at relatively lower power levels, such that the total pump power is kept the
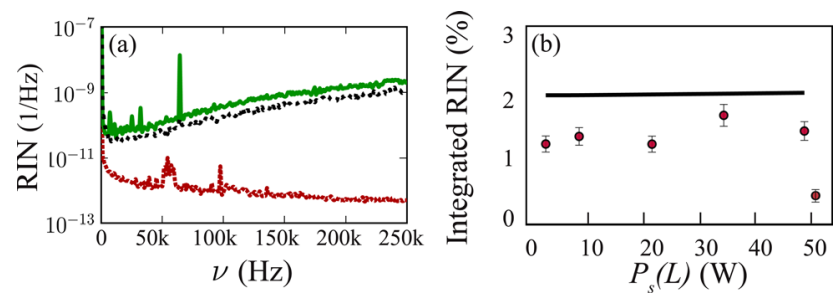

FIG. 4. (a) Measured RIN spectra of the pump (red-densely dotted curve), amplifier output (green-solid curve), and calculated RIN spectra of amplifier output (black-loosely dotted curve) for cladding-pumped amplifier. (b) Measured (dots) and calculated (solid curve) integrated RIN values as a function of signal power for high power double-clad amplifier. 


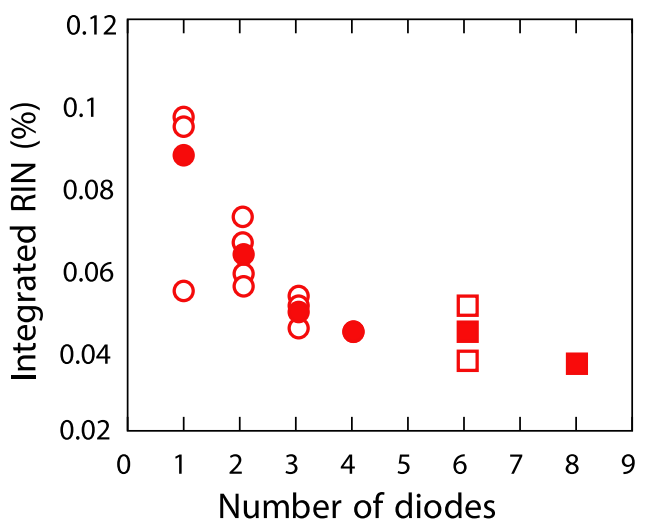

FIG. 5. Measured integrated RIN of multi-mode diode combinations (open symbols) and their mean values (filled symbols). Circles denote measurements for which each diode was driven by a different diode driver and squares denote measurements for which pairs of diodes had to be connected to one diode driver, resulting in possibly partially common fluctuations.

same in each case. In case of multiple pump sources, they were thermally decoupled from each other and they were driven by multiple electronic drivers. This way, we ensure that their noise fluctuations were largely independent of each other. The measurements were performed for multiple sets of diodes formed out of a set of 8 diodes. We observe that the average integrated RIN (filled symbols, Fig. 5) of the combination of diodes decreases approximately with inverse square root of number of diodes combined, which is to be expected in the limit of completely independent noise for each diode. The squares in the figure correspond to measurements taken with possibly correlated noise sources due to having had to connect more than one diode to each diode driver, as we did not have enough diode drivers at hand. It appears that it is best to pump an amplifier with a relatively large number of independently driven, lower-power pump diodes than a single, high-power pump diode.

In summary, we have experimentally characterized the transfer of seed and pump modulations to the output of a fiber amplifier, supported by a simple, yet effective analytical model. Our results address a practical need, which is to predict the intensity noise of an amplifier, obtaining excellent agreement with experiments. In the future, we plan to apply the modulation transfer approach to a laser oscillator, which is expected to lead to qualitatively different results due to the feedback provided by the cavity. We have further shown that using a combination of multiple diodes running at lower powers instead of a single diode running at high power results in a lower noise profile for given total pump power. Using our practical approach, experimental development of low-noise fiber laser amplifiers can be guided by theory with high accuracy.

This work was supported by the Scientific and Technological Research Council of Turkey (TÜBITAK) (Grant No. 112T944).

${ }^{1}$ L. Budunoğlu, C. Ülgüdür, B. Oktem, and F. Ö. Ilday, 34, Opt. Lett. 2516-2518 (2009).

${ }^{2}$ P. K. Mukhopadhyay, K. Özgören, I. L. Budunoğlu, and F. Ö. Ilday, IEEE J. Sel. Top. Quantum Electron. 15, 145-152 (2009).

${ }^{3}$ N. R. Newbury and W. C. Swann, J. Opt. Soc. Am. B 24, 1756-1770 (2007).

${ }^{4}$ S. Novak and A. Moesle, J. Lightwave Technol. 20, 975-985 (2002).

${ }^{5}$ J. Freeman and J. Conradi, IEEE Photonics Technol. Lett. 5, 224-226 (1993).

${ }^{6}$ M. Tröbs, P. Wessels, and C. Fallnich, Opt. Express 13, 2224-2235 (2005).

${ }^{7}$ B. Oktem, C. Ülgüdür, and F. Ö. Ilday, Nat. Photonics 4, 307-311 (2010).

${ }^{8}$ C. R. Giles and E. Desurvire, J. Lightwave Technol. 9, 271-283 (1991).

${ }^{9}$ G. P. Agrawal, Fiber-Optics Communication Systems (John Wiley \& Sons, Inc., 2009).

${ }^{10}$ F. Ö. Ilday, J. Buckley, and F. W. Wise, Phys. Rev. Lett. 92, 213902 (2004).

${ }^{11}$ F. Ö. Ilday, H. Lim, J. R. Buckley, and F. W. Wise, Opt. Lett. 28, 1362-1364 (2003).

${ }^{12}$ T. D. Mulder, R. P. Scott, and B. H. Kolner, Opt. Express 16, 14186-14191 (2008).

${ }^{13}$ H. Hodara, Proc. IEEE 53, 696-704 (1965).

${ }^{14}$ P. Elahi, S. Ylmaz, Ö. Akçaalan, H. Kalaycioğlu, B. Öktem, Ç. Şenel, F. Ö. Ilday, and K. Eken, Opt. Lett. 37, 3042-3044 (2012). 